Symmetry, Integrability and Geometry: Methods and Applications

SIGMA * $\left(201^{*}\right), * * *, 20$ pages

\title{
Total Rarita-Schwinger operators in Clifford analysis
}

\author{
David EELBODE ${ }^{\dagger}$ and Peter VAN LANCKER $\ddagger$ \\ $\dagger$ University of Antwerp, Department of Mathematics \\ Campus Middelheim, G-Building, Middelheimlaan 1, 2020 Antwerpen (Belgium) \\ E-mail: david.eelbode@ua.ac.be \\ $\ddagger$ University College Ghent (Member of Ghent University Association) \\ Department of Engineering Sciences \\ Schoonmeersstraat 52, 9000 Ghent (Belgium) \\ E-mail: Peter.VanLancker@hogent.be
}

Received ???, in final form ????; Published online ????

doi:10.3842/SIGMA.201*.***

\begin{abstract}
Rarita-Schwinger operators in Clifford analysis can be realized as first-order differential operators acting on functions $f(x ; u)$ taking values in the vector space of monogenic polynomials $\mathcal{M}_{k}\left(\mathbb{R}^{m}\right)$, where $k \in \mathbb{N}$ is fixed. In this paper, the Scasimir operator for the Lie superalgebra $\mathfrak{o s p}(1 \mid 2)$ will be used to construct an invariant operator which acts on the full space of functions in two vector variables and therefore has more invariance properties. Also the fundamental solution for this operator will be constructed.
\end{abstract}

Key words: Rarita-Schwinger operator; Clifford analysis; fundamental solution

2010 Mathematics Subject Classification: 42B99; 47B38; $35 \mathrm{Q} 70$

\section{Introduction}

Classical Clifford analysis is usually defined as a function theory generalizing complex analysis to the case of arbitrary dimension $m \in \mathbb{N}$ (considered as a formal parameter), and at the same time refining classical harmonic analysis in $\mathbb{R}^{m}$. Indeed, a well-known realization for the Lie algebra $\mathfrak{s l}(2)$ in terms of (scalar) operators on $\mathbb{R}^{m}$ is given by

$$
\mathfrak{s l}(2) \cong \operatorname{Alg}\left\{-\frac{1}{2} \Delta_{x}, \frac{1}{2}|x|^{2}, \mathbb{E}_{x}+\frac{m}{2}\right\},
$$

where $\Delta_{x}$ denotes the Laplace operator, $|x|^{2}=\sum_{j} x_{j}^{2}$ and $\mathbb{E}_{x}$ is the Euler operator measuring the degree of homogeneity in $\left(x_{1}, \cdots, x_{m}\right)$. In Clifford analysis, one adds the vector variable $x$ and the Dirac operator $\partial_{x}$, the latter being the unique (up to a constant) conformally invariant differential operator acting on spinor-valued (or Clifford algebra-valued) functions. This then leads to an operator realization for the Lie superalgebra

$$
\mathfrak{o s p}(1 \mid 2) \cong \operatorname{Alg}\left\{\frac{i \sqrt{2}}{2} x,-i \frac{\sqrt{2}}{2} \partial_{x}\right\},
$$

whose even part is the Lie algebra $\mathfrak{s l}(2)$. The constants are hereby choosen in such a way that one obtains the classical (anti-)commutation relations, for which we refer to [12]. This Dirac operator $\partial_{x}$, generalizing the Cauchy-Riemann operator in complex analysis and factorizing the Laplacian $\Delta_{x}$, is the main object of study in classical Clifford analysis. We refer the reader to $[3,8,13]$ or to the overview paper [7] for a general introduction to this branch of classical analysis. Since a few years, Clifford analysis turned out to be an elegant framework to study function theoretical problems not only for the classical Dirac operator acting on spinor-valued functions, but also 
D. Eelbode and P. Van Lancker

for far-reaching generalizations of it, acting on functions which take their values in arbitrary half-integer irreducible spin-representations. The earliest results involved generalizations of the Rarita-Schwinger operator $(k=1)$, again inspired by equations coming from theoretical physics: in a series of papers $[4,5,17]$, one can find a collection of function theoretical fundaments for these operators acting on functions taking values in irreducible modules with highest weight $\left(k+\frac{1}{2}, \frac{1}{2}, \cdots, \frac{1}{2}\right)$, with $k \in \mathbb{N}$. These modules are then realized as spaces of homogeneous polynomial solutions for the Dirac operator $\partial_{u}$ in a dummy variable $u \in \mathbb{R}^{m}$. In this paper, we will show how one can use the Scasimir operator, see $[2,12]$, to construct an invariant operator $\mathcal{R}_{s}$ acting on the full space of polynomials in the dummy variable. This operator will thus satisfy additional symmetry properties.

The paper is organized as follows. After an introduction to Clifford analysis in section 2, we will introduce in section 3 and 4 the Scasimir operator $S c_{1}$ and corresponding Hilbert operator $H$ for $\mathfrak{o s p}(1 \mid 2)$. The Hilbert operator $H$ plays a fundamental role in defining the total Rarita-Schwinger operator $\mathcal{R}$ and total twistor operator $\mathcal{T}$. These operators incorporate the infinite number $(k \in \mathbb{N})$ of fundamental operators defined in $[4,5,16]$ into two basic operators, thus leading to a more unified and algebraically more transparent approach (section 5,6). A particular rescaling of the total Rarita-Schwinger operator $\mathcal{R}$ leads to the construction of the so-called rescaled total Rarita-Schwinger operator $\mathcal{R}_{s}$ which commutes with the full algebra osp $(1 \mid 2)$ and hence enjoys more invariance properties than the classical operators (section 7 ). Its behaviour under the action of the conformal group and a construction of the fundamental solution based on Riesz potentials will be discussed in section 8 and 9 . In the last section we derive an integral representation for the Hilbert operator $H$.

Finally, the technique of using Scasimir operators to construct higher spin Dirac operators can be done for more general cases than the one that will be treated in the present paper. We will elaborate this in an upcoming paper.

\section{Clifford Analysis}

Let $\left(e_{1}, \ldots, e_{m}\right)$ be an orthonormal basis for the Euclidean vector space $\mathbb{R}^{m}$, and denote by $\mathbb{R}_{m}$ the real Clifford algebra generated by these basis elements together with the defining relations $e_{i} e_{j}+e_{j} e_{i}=-2 \delta_{i j}(i, j=1, \ldots, m)$. The complex Clifford algebra $\mathbb{C}_{m}$ is then defined as $\mathbb{C}_{m}:=\mathbb{R}_{m} \otimes_{\mathbb{R}} \mathbb{C}$. Any element $x$ of $\mathbb{R}^{m}$ can be embedded inside this Clifford algebra, by means of $x \hookrightarrow \sum e_{j} x_{j}$. The classical inner and outer (wedge) product of two vectors $x, y \in \mathbb{R}^{m}$ can then be defined in terms of the Clifford product:

$$
\langle x, y\rangle:=-\frac{1}{2}(x y+y x) \text { and } x \wedge y:=\frac{1}{2}(x y-y x) .
$$

Inside the complex Clifford algebra $\mathbb{C}_{m}$, one can realize the spinor space(s) $\mathbb{S}^{ \pm}$as a minimal left ideal, using a primitive idempotent. Note that the parity of spinors only needs to be taken into account in case of even dimensions $m=2 n$ (in odd dimensions $m=2 n+1$, it suffices to disregard the \pm ). The vector space(s) $\mathbb{S}^{ \pm}$define(s) the basic half-integer representation(s) for the spin group $\operatorname{Spin}(m)$, described by the highest weight $\left(\frac{1}{2}, \frac{1}{2}, \cdots, \pm \frac{1}{2}\right)$ under the multiplicative left action $\psi \mapsto s \psi$ for all $\psi \in \mathbb{S}$ and $s \in \operatorname{Spin}(m)$. Note that the spin group itself can also be realized inside the Clifford algebra:

$$
\operatorname{Spin}(m)=\left\{s=\prod_{j=1}^{2 k} s_{j}: k \in \mathbb{N}, s_{j} \in S^{m-1}\right\},
$$

where $S^{m-1} \subset \mathbb{R}^{m}$ denotes the unit sphere. The classical Dirac operator in $\mathbb{R}^{m}$ is then given by $\partial_{x}=\sum_{j} e_{j} \partial_{x_{j}}$. This is the unique, conformally invariant first order differential operator 
acting on $\mathbb{S}^{ \pm}$-valued functions $f(x)$ on $\mathbb{R}^{m}$. Note that whereas this operator maps $\psi(x)$ in $\mathbb{S}^{ \pm}$ to $\partial_{x} \psi(x) \in \mathbb{S}^{\mp}$, in Clifford analysis one often considers functions taking values in the whole Clifford algebra $\mathbb{C}_{m}$, which can be seen as a direct sum of several equivalent irreducible spinor representations. As this does not affect the function theoretical result under consideration, we will often consider functions $f(x)$ taking values in the space $V$, which may then stand for either the spinor space(s) or the whole Clifford algebra. A $V$-valued function $f$ is called monogenic in $x$ if it satisfies the equation $\partial_{x} f=0$. Since $\partial_{x}^{2}=-\triangle_{x}$, monogenic functions on $\mathbb{R}^{m}$ are a refinement of harmonic functions. We fix the following notations: the space of $V$-valued polynomials in $\mathbb{R}^{m}$ is denoted by $\mathcal{P}\left(\mathbb{R}^{m}, V\right)$ and

$$
\begin{aligned}
\mathcal{H}\left(\mathbb{R}^{m}, V\right) & =\left\{P(x) \in \mathcal{P}\left(\mathbb{R}^{m}, V\right): \triangle_{x} P(x)=0\right\} \\
\mathcal{M}\left(\mathbb{R}^{m}, V\right) & =\left\{P(x) \in \mathcal{P}\left(\mathbb{R}^{m}, V\right): \partial_{x} P(x)=0\right\} .
\end{aligned}
$$

The corresponding subspaces of $k$-homogeneous polynomials are denoted by means of an extra subscript $k$. The spaces $\mathcal{M}_{k}\left(\mathbb{R}^{m}, V\right)$ and $\mathcal{H}_{k}\left(\mathbb{R}^{m}, V\right)$ are known as the spaces of (inner) spherical monogenics and harmonics of order $k$ which are of major importance in harmonic analysis related to $\mathrm{SO}(m)$ or its double cover $\operatorname{Spin}(m)$. Not only the spinor space(s) $\mathbb{S}^{ \pm}$, but also other halfinteger $\operatorname{Spin}(m)$-representations can be characterized using the language of Clifford algebras and Clifford analysis, see e.g. [6]. For our purposes, it suffices to note that the space $\mathcal{M}_{k}\left(\mathbb{R}^{m}, \mathbb{S}^{ \pm}\right)$ in a dummy variable $u \in \mathbb{R}^{m}$ defines a model for the irreducible $\operatorname{Spin}(m)$-representation with highest weight $\left(k+\frac{1}{2}, \frac{1}{2}, \cdots, \frac{1}{2}, \pm \frac{1}{2}\right)$, under the left regular representation (or $L$-representation) $L(s) P(u):=s P(\bar{s} u s), s \in \operatorname{Spin}(m)$. Hereby, ${ }^{-}$denotes the Clifford conjugation which is the antiinvolution determined by $\bar{e}_{i}=-e_{i}$. Also here, we will often speak about the spaces $\mathcal{M}_{k}\left(\mathbb{R}^{m}, V\right)$ which reduce to a direct sum of equivalent modules in case $V=\mathbb{C}_{m}$.

The $\Gamma$-operator in Clifford analysis is traditionally introduced as follows: let $(r, \omega) \in \mathbb{R}_{+} \times S^{m-1}$ be spherical coordinates on $\mathbb{R}^{m}$. The Dirac operator can be expressed as $\partial_{x}=\omega\left(\partial_{r}+\frac{1}{r} \Gamma_{x}\right)$ where the angular operator $\Gamma_{x}$ is the bivector-valued differential operator given by

$$
\Gamma_{x}:=-\sum_{i<j} e_{i j}\left(x_{i} \partial_{x_{j}}-x_{j} \partial_{x_{i}}\right)=-\sum_{i<j} e_{i j} L_{i j}
$$

The (axial momentum) operators $L_{i j}:=x_{i} \partial_{x_{j}}-x_{j} \partial_{x_{i}}$ are the usual generators of the Lie algebra $\mathfrak{s o}(m)$ obtained by deriving the left regular representation $h(s) P(u):=P(\bar{s} u s)$, for all $s \in \operatorname{Spin}(m)$.

As was mentioned in the introduction, the Dirac operator $\partial_{x}$ and the vector variable $x$ generate the Lie superalgebra $\mathfrak{o s p}(1 \mid 2)$, which is a concise way to summarize the most crucial operator identities in Clifford analysis. Apart from its geometric significance as a Dirac-type operator on the sphere, $\Gamma_{x}$ appears also in a slightly different form as a certain element of $\mathcal{U}(\mathfrak{o s p}(1 \mid 2))$; a simple computation shows that $\Gamma_{x}$ can be expressed as

$$
\Gamma_{x}=\frac{1}{2}\left(\left[x, \partial_{x}\right]+m\right)=-\mathbb{E}_{x}-x \partial_{x} .
$$

Precisely these type of identities reveal a close relationship between $\Gamma_{x}$ and a rather special uniquely defined element of $\mathcal{U}(\mathfrak{o s p}(1 \mid 2))$, the so-called Scasimir operator $S c_{1}$ of $\mathfrak{o s p}(1 \mid 2)$, which will be introduced in the next section.

\section{The Scasimir operator $S c_{1}$ for $\mathfrak{o s p}(1 \mid 2)$}

In [2], the authors introduced the Scasimir operator for the algebra osp $(1 \mid 2 p)$. As opposed to the Casimir operator $C_{2}(L)$ for $\mathfrak{o s p}(1 \mid 2 p)$, the operator $S c_{p}$ anti-commutes with the odd generators (and hence commutes with all the even generators). Its square $S c_{p}^{2}$ commutes with the odd 
generators, which means that the Scasimir operator $S c_{p}$ can be interpreted as a "square root" of a Casimir operator. In case of one vector variable $(p=1)$, this operator is explicitly given (in our polynomial model) by a shift of the anti-commutator of the odd generators (from now on in the dummy variable $\left.u \in \mathbb{R}^{m}\right)$ :

$$
S c_{1}:=\frac{1}{2}\left(u \partial_{u}-\partial_{u} u\right)-\frac{1}{2}=\frac{m-1}{2}-\Gamma_{u} \in \mathcal{U}(\mathfrak{o s p}(1 \mid 2)) .
$$

Using the defining relations for $\mathfrak{o s p}(1 \mid 2)$, one can easily verify by explicit calculations that the relations $\left\{S c_{1}, u\right\}=\left\{S c_{1}, \partial_{u}\right\}=0$ hold. The Casimir operators of the $h$ and $L$-representation can then be expressed as polynomials in $\Gamma_{u}$ (see [8]):

$$
\begin{aligned}
C_{2}(h) & :=\sum_{i<j} L_{i j}^{2}=\Gamma_{u}\left(m-2-\Gamma_{u}\right) \\
C_{2}(L) & :=\sum_{i<j}\left(L_{i j}-\frac{1}{2} e_{i j}\right)^{2}=\Gamma_{u}\left(m-1-\Gamma_{u}\right)-\frac{m(m-1)}{8} .
\end{aligned}
$$

The Casimir operator $C_{2}(L)$ is thus related to the Scasimir operator $S c_{1}$ :

$$
C_{2}(L)=-S c_{1}^{2}+\frac{(m-1)(m-2)}{8} .
$$

The Casimir operator $C_{2}(h)$ is also known in literature as the Laplace-Beltrami operator on the unit sphere $S^{m-1}$. The action of $S c_{1}$ on $\mathcal{P}\left(\mathbb{R}^{m}, V\right)$ is most easily understood in terms of the monogenic Fischer decomposition of spinor-valued polynomials. First of all, the classical Howe duality for $\mathrm{SO}(m) \times \mathfrak{s l}(2)$ tells us that

$$
\mathcal{P}\left(\mathbb{R}^{m}, \mathbb{C}\right)=\bigoplus_{k \geq 0} \mathbb{V}_{k}^{\infty} \otimes \mathcal{H}_{k}\left(\mathbb{R}^{m}, \mathbb{C}\right)
$$

where $\mathbb{V}_{k}^{\infty}$ denotes a lowest-weight (infinite-dimensional) irreducible Verma module for the Lie algebra $\mathfrak{s l}(2)$. Making use of the Fischer decomposition for $V$-valued harmonic polynomials into monogenics, given by

$$
\mathcal{H}_{k}\left(\mathbb{R}^{m}, V\right)=\mathcal{M}_{k}\left(\mathbb{R}^{m}, V\right) \oplus\left(1-\delta_{k, 0}\right) u \mathcal{M}_{k-1}\left(\mathbb{R}^{m}, V\right)
$$

we can refine this result to

$$
\begin{aligned}
\mathcal{P}\left(\mathbb{R}^{m}, V\right) & =\bigoplus_{k \geq 0} \mathbb{V}_{k}^{\infty} \otimes\left(\mathcal{M}_{k}\left(\mathbb{R}^{m}, V\right) \oplus\left(1-\delta_{k, 0}\right) u \mathcal{M}_{k-1}\left(\mathbb{R}^{m}, V\right)\right) \\
& =\bigoplus_{k \geq 0}\left(\mathbb{V}_{k}^{\infty} \oplus u \mathbb{V}_{k+1}^{\infty}\right) \otimes \mathcal{M}_{k}\left(\mathbb{R}^{m}, V\right) \\
& =\bigoplus_{k \geq 0} \mathbb{W}_{k}^{\infty} \otimes \mathcal{M}_{k}\left(\mathbb{R}^{m}, V\right) .
\end{aligned}
$$

Here, $\mathbb{W}_{k}^{\infty}$ denotes a lowest-weight (infinite-dimensional) irreducible Verma module for the Lie superalgebra osp $(1 \mid 2)$. Note that (3) corresponds to the decomposition of $\mathcal{P}\left(\mathbb{R}^{m}, V\right)$ under the Howe dual pair $\operatorname{Spin}(m) \times \mathfrak{o s p}(1 \mid 2)$. This is the symmetry behind the full monogenic Fischer decomposition, a result which is of fundamental importance in Clifford analysis of functions of one vector variable. The operator $S c_{1}$ anti-commutes with $u$ and $\partial_{u}$, hence its eigenvalue-analysis on $\mathcal{P}\left(\mathbb{R}^{m}, V\right)$ is reduced to:

\begin{tabular}{|c|c|c|}
\hline & $\mathcal{M}_{k}$ & $u \mathcal{M}_{k}$ \\
\hline$S c_{1}$ & $+\left(k+\frac{m-1}{2}\right)$ & $-\left(k+\frac{m-1}{2}\right)$ \\
\hline
\end{tabular}




\section{The Hilbert operator $H$ of $\mathfrak{o s p}(1 \mid 2)$}

The square of the Scasimir operator being a positive operator, we can also define its square root $\left|S c_{1}\right|$. Its definition easily follows from the spectral decomposition of $S c_{1}^{2}$, where ${ }^{\dagger}$ denotes the adjoint for the Fischer inner product (cf. infra, definition 7):

$$
\left|S c_{1}\right|:=\left(S c_{1} S c_{1}^{\dagger}\right)^{\frac{1}{2}}=\left(S c_{1}^{2}\right)^{\frac{1}{2}}
$$

The operator $\left|S c_{1}\right|$, which will also commute with all the $\mathfrak{o s p}(1 \mid 2)$-generators, can now be used to define a Hilbert-type operator $H$ on $\mathcal{P}\left(\mathbb{R}^{m}, V\right)$ :

\section{Definition 1.}

$$
H:=\frac{S c_{1}}{\left|S c_{1}\right|}=\frac{m-1-2 \Gamma_{u}}{\left|m-1-2 \Gamma_{u}\right|}=\operatorname{sgn}\left(S c_{1}\right)
$$

Inspection of its eigenvalues shows that the operator $\left|S c_{1}\right|$ is invertible. The notation division by $\left|S c_{1}\right|$ is to be understood as multiplication with $\left|S c_{1}\right|^{-1}$ (left or right multiplication yields the same result). We attribute the name Hilbert to this operator because $H$ can be seen as a canonical extension of the spherical Hilbert operator $H_{S}$ which is traditionally defined and studied within the framework of square integrable functions on the sphere, see e.g. [8]:

Definition 2. The operator $H_{S}$ is defined on $L^{2}\left(S^{m-1}\right)$ by means of

$$
\left(H_{S} f\right)(\omega):=\frac{1}{A_{m}} \mathrm{PV} \int_{S^{m-1}} \frac{1+\omega \xi}{|1+\omega \xi|^{m}} f(\xi) d \xi
$$

where ' $P V$ ' refers to the principal value of the singular integral operator under consideration.

The operator $H_{S}$ has eigenvalues \pm 1 and the corresponding eigenspaces are the spaces of inner and outer spherical monogenics: $H_{S}[\mathcal{M}]=\mathcal{M}$ and $H_{S}[\xi \mathcal{M}]=-\omega \mathcal{M}$. Initially, $H_{S}$ is defined on the subspace $\mathcal{H}\left(\mathbb{R}^{m}, V\right)$, with

$$
\mathcal{M}\left(\mathbb{R}^{m}, V\right) \oplus \xi \mathcal{M}\left(\mathbb{R}^{m}, V\right)=\mathcal{H}\left(\mathbb{R}^{m}, V\right) \subset \mathcal{P}\left(\mathbb{R}^{m}, V\right) .
$$

If one considers $\mathcal{P}\left(\mathbb{R}^{m}, V\right)$ as an $\mathrm{SO}(m) \times \mathfrak{s l}(2)$-module, it is clear that $H$, as defined in (4), is precisely the $\mathfrak{s l}(2)$-invariant extension of $H_{S}$ to $\mathcal{P}\left(\mathbb{R}^{m}, V\right)$. However, the symmetry properties of the $H$-operator are better understood by regarding $\mathcal{P}\left(\mathbb{R}^{m}, V\right)$ as a $\operatorname{Spin}(m) \times \mathfrak{o s p}(1 \mid 2)$-module. For this action, $H$ is the unique operator which acts like the identity on the direct sum $\mathcal{M}\left(\mathbb{R}^{m}, V\right)$ of all lowest weight modules and (super-)commutes with the $\mathfrak{o s p}(1 \mid 2)$-factor:

$$
\{H, u\}=\left\{H, \partial_{u}\right\}=\left[H,|u|^{2}\right]=\left[H, \Delta_{u}\right]=\left[H, \mathbb{E}_{u}+\frac{m}{2}\right]=0 .
$$

At the end of the paper we will give an integral representation of $H$. Note that there also exists an alternative definition for this Hilbert operator $H$ in terms of a shifted Scasimir operator $P_{1}$ defined as

$$
P_{1}:=S c_{1}-\frac{1}{2}=\frac{m-2}{2}-\Gamma_{u} .
$$

This operator commutes with the even part $\mathfrak{s l}(2) \cong\left\{\Delta_{u},|u|^{2}, \mathbb{E}_{u}+\frac{m}{2}\right\}$ of $\mathfrak{o s p}(1 \mid 2)$ and appears naturally as the operator in $\mathcal{U}(\mathfrak{o s p}(1 \mid 2))$ for which

$$
P_{1}^{2}=-C_{2}(h)+\left(\frac{m-2}{2}\right)^{2} .
$$


D. Eelbode and P. Van Lancker

This relation shows that the operator $P_{1}$ (opposed to $S c_{1}$ ) behaves better on $V$-valued spherical harmonics $\mathcal{H}_{k}\left(\mathbb{R}^{m}, V\right)$ of order $k$, as can be seen from its eigenvalues (remark the shift $k \rightarrow k-1$ in the second part):

\begin{tabular}{|c|c|c|}
\hline & $\mathcal{M}_{k}$ & $u \mathcal{M}_{k-1}$ \\
\hline$P_{1}$ & $+\left(k+\frac{m-2}{2}\right)$ & $-\left(k+\frac{m-2}{2}\right)$ \\
\hline
\end{tabular}

Whereas this operator $P_{1}$ does not anti-commute with the odd generators, its square $P_{1}^{2}$ is a positive operator commuting with the even generators. We can therefore again define a square root operator of $P_{1}^{2}$, denoted by means of $\left|P_{1}\right|$, which also commutes with the even generators. Despite the fact that neither $P_{1}$ nor its absolute value $\left|P_{1}\right|$ anti-commute with the odd generators of $\mathfrak{o s p}(1 \mid 2)$, their quotient still behaves in a nice way. As a matter of fact, $H$ can be represented alternatively as follows:

\section{Proposition 1.}

$$
H=\operatorname{sgn}\left(S c_{1}\right)=\operatorname{sgn}\left(P_{1}\right)=\frac{P_{1}}{\left|P_{1}\right|}=\frac{m-2-2 \Gamma_{u}}{\left|m-2-2 \Gamma_{u}\right|} .
$$

Proof. Since both $\operatorname{sgn}\left(S c_{1}\right)$ and $\operatorname{sgn}\left(P_{1}\right)$ commute with the even generators of $\mathfrak{o s p}(1 \mid 2)$, it is enough to verify this statement on $V$-valued harmonics. It then suffices to note that the operators $S c_{1},\left|S c_{1}\right|, P_{1},\left|P_{1}\right|$ acts diagonally on each of the summands in (2).

\section{The total Rarita-Schwinger and twistor operator}

Recall that $H$ (as the signum operator of the Scasimir) defines an involution on $\mathcal{P}\left(\mathbb{R}^{m}, V\right)$. Accordingly we can define a $\operatorname{Spin}(m)$-invariant $\mathbb{Z}_{2}$-grading:

$$
\mathcal{P}\left(\mathbb{R}^{m}, V\right)=\mathcal{P}_{0}\left(\mathbb{R}^{m}, V\right) \oplus \mathcal{P}_{1}\left(\mathbb{R}^{m}, V\right)
$$

Hereby the (even) 0-part and the (odd) 1-part are identified with respectively the $(+1)$ and (-1)-eigenspaces of $H$ :

$$
\mathcal{P}_{0}\left(\mathbb{R}^{m}, V\right)=\bigoplus_{l \geq 0}|u|^{2 l} \mathcal{M}\left(\mathbb{R}^{m}, V\right) \text { and } \mathcal{P}_{1}\left(\mathbb{R}^{m}, V\right)=\bigoplus_{l \geq 0} u^{2 l+1} \mathcal{M}\left(\mathbb{R}^{m}, V\right) .
$$

Let $a \in \mathbb{C}_{m}$ be a (constant) Clifford number which acts by left multiplication on $f \in \mathcal{P}\left(\mathbb{R}^{m}, V\right)$. Consider the decomposition $a f=\check{\pi}_{0}(a) f+\check{\pi}_{1}(a) f$ where the projections on the even and odd part are given by

$$
\check{\pi}_{0}(a) f:=\frac{1}{2}(a+H a H) f \quad \text { and } \quad \check{\pi}_{1}(a) f:=\frac{1}{2}(a-H a H) f .
$$

Clearly the operator $H$ commutes with $\check{\pi}_{0}(a)$ and anti-commutes with $\check{\pi}_{1}(a)$. If we now consider the $\mathbb{Z}_{2}$-grading on $\mathcal{P}\left(\mathbb{R}^{m}, V\right)$ defined by $H$, then $\check{\pi}_{0}(a)$ and $\check{\pi}_{1}(a)$ are the even and odd part of the operator defined by $a \in$ End $\left(\mathcal{P}\left(\mathbb{R}^{m}, V\right)\right)$.

Instead of working with polynomials $f(x ; u) \in \mathcal{P}\left(\mathbb{R}^{m}, \mathcal{M}_{k}\right)$ or $\mathcal{P}\left(\mathbb{R}^{m}, \mathcal{M}\right)$ we will from now on often consider $f(x ; u) \in \mathcal{P}\left(\mathbb{R}^{m}, \mathbb{C}\right) \otimes \mathcal{P}\left(\mathbb{R}^{m}, V\right)$, which we will identify with $\mathcal{P}\left(\mathbb{R}^{2 \times m}, V\right)$. The asymmetry in the notation $f(x ; u)$ then emphasizes the difference between the domain variable $x$ and the dummy variable $u$ which is used to describe the values of the map $x \mapsto f(x ; u)$. The decomposition of the action of the Dirac operator $\partial_{x}$ on $f(x ; u)$ in $\mathcal{P}\left(\mathbb{R}^{2 \times m}, V\right)$ in its even and odd part then leads to the following: 
Definition 3. The total Rarita-Schwinger operator $\mathcal{R}$ is defined to be the even operator $\check{\pi}_{0}\left(\partial_{x}\right)$ :

$$
\mathcal{R}:=\frac{1}{2}\left(\partial_{x}+H \partial_{x} H\right)
$$

The total twistor operator $\mathcal{T}$ is defined to be the odd operator $\check{\pi}_{1}\left(\partial_{x}\right)$ :

$$
\mathcal{T}:=\frac{1}{2}\left(\partial_{x}-H \partial_{x} H\right)
$$

where both operators are considered as endomorphisms on $\mathcal{P}\left(\mathbb{R}^{2 \times m}, V\right)$.

Proposition 2. The total $R S$ - and twistor operator, considered as endomorphisms on $\mathcal{P}\left(\mathbb{R}^{2 \times m}, V\right)$, can be expressed as

$$
\begin{aligned}
& \mathcal{R}=\frac{1}{2 P_{1}}\left\{P_{1}, \partial_{x}\right\}=\frac{1}{2\left(m-2-2 \Gamma_{u}\right)}\left\{m-2-2 \Gamma_{u}, \partial_{x}\right\} \\
& \mathcal{T}=\frac{1}{2 P_{1}}\left[P_{1}, \partial_{x}\right]=\frac{1}{2\left(m-2-2 \Gamma_{u}\right)}\left[m-2-2 \Gamma_{u}, \partial_{x}\right],
\end{aligned}
$$

and both operators commute with the even part $\mathfrak{s l}(2) \cong\left\{\Delta_{u},|u|^{2}, \mathbb{E}_{u}+\frac{m}{2}\right\}$ of $\mathfrak{o s p}(1 \mid 2)$.

Proof. Recalling the alternative definition of the Hilbert operator

$$
H=\frac{P_{1}}{\left|P_{1}\right|}=\frac{m-2-2 \Gamma_{u}}{\left|m-2-2 \Gamma_{u}\right|},
$$

the first statement follows by substituting this expression into definition 3 and observing that $\left|P_{1}\right|$ commutes with $\partial_{x}$. Note that this heavily relies on the definition of $H$ in terms of $P_{1}$. Indeed, since $P_{1}^{2}$ is a scalar-valued element of $\mathcal{U}(\mathfrak{o s p}(1 \mid 2))$, it is clear that $\left[P_{1}^{2}, \partial_{x}\right]=0$ and this ensures that $\left[\left|P_{1}\right|, \partial_{x}\right]=0$. The second property follows from the fact that both $P_{1}$ and $\partial_{x}$ commute with $\mathfrak{s l}(2)$.

\section{Operators on $\mathcal{P}\left(\mathbb{R}^{m}, \mathcal{M}_{k}\right)$ and $\mathcal{P}\left(\mathbb{R}^{m}, u \mathcal{M}_{k-1}\right)$}

The construction of the (generalized) Rarita-Schwinger operator $\mathcal{R}_{k}$, see e.g. $[4,5,16]$, is based on the notion of monogenic operators. This relies on the following property: for any $a \in \mathbb{C}_{m}$ and $P_{k} \in \mathcal{M}_{k}\left(\mathbb{R}^{m}, V\right)$, we have that $a P_{k} \in \mathcal{H}_{k}\left(\mathbb{R}^{m}, V\right)$. Using the Fischer decomposition of spherical harmonics into spherical monogenics, we then get:

$$
a P_{k}(u)=\left(\frac{k+m-2-\Gamma_{u}}{2 k+m-2}\right) a P_{k}(u)+\left(\frac{k+\Gamma_{u}}{2 k+m-2}\right) a P_{k}(u) .
$$

In this way the decomposition of the left multiplication operator $a: \mathcal{M}_{k}\left(\mathbb{R}^{m}, V\right) \rightarrow \mathcal{H}_{k}\left(\mathbb{R}^{m}, V\right)$ : $P_{k} \mapsto a P_{k}$ as the sum of two (monogenic) operators is obtained. In case $a=\partial_{x}$, these operators are usually referred to as the (generalized) Rarita-Schwinger operator $\mathcal{R}_{k}$ and dual twistor operator $\mathcal{T}_{k}{ }^{*}$. Together with the decomposition of the left multiplication operator $a$ acting on $u P_{k-1}(u)$, one obtains four operators $\mathcal{R}_{k}, \mathcal{D}_{k-1}, \mathcal{T}_{k}, \mathcal{T}_{k}{ }^{*}$ which admit a simple form in terms of the Hilbert operator $H$ :

1. The Rarita-Schwinger operator $\mathcal{R}_{k}: \mathcal{P}\left(\mathbb{R}^{m}, \mathcal{M}_{k}\right) \rightarrow \mathcal{P}\left(\mathbb{R}^{m}, \mathcal{M}_{k}\right)$

$$
\begin{aligned}
\mathcal{R}_{k} f(x ; u) & =\left(\frac{k+m-2-\Gamma_{u}}{2 k+m-2}\right) \partial_{x} f(x ; u) \\
& =\frac{1}{2}(1+H) \partial_{x} f(x ; u), \quad f(x ; u) \in \mathcal{P}\left(\mathbb{R}^{m}, \mathcal{M}_{k}\right)
\end{aligned}
$$


2. The equivalent operator $\mathcal{D}_{k-1}: \mathcal{P}\left(\mathbb{R}^{m}, u \mathcal{M}_{k-1}\right) \rightarrow \mathcal{P}\left(\mathbb{R}^{m}, u \mathcal{M}_{k-1}\right)$

$$
\begin{aligned}
\mathcal{D}_{k-1} u f(x ; u) & =\left(\frac{k+\Gamma_{u}}{2 k+m-2}\right) \partial_{x} u f(x ; u) \\
& =\frac{1}{2}(1-H) \partial_{x} u f(x ; u), \quad f(x ; u) \in \mathcal{P}\left(\mathbb{R}^{m}, \mathcal{M}_{k-1}\right) .
\end{aligned}
$$

3. The twistor operator $\mathcal{T}_{k}: \mathcal{P}\left(\mathbb{R}^{m}, u \mathcal{M}_{k-1}\right) \rightarrow \mathcal{P}\left(\mathbb{R}^{m}, \mathcal{M}_{k}\right)$

$$
\begin{aligned}
\mathcal{T}_{k} u f(x ; u) & =\left(\frac{k+m-2-\Gamma_{u}}{2 k+m-2}\right) \partial_{x} u f(x ; u) \\
& =\frac{1}{2}(1-H) \partial_{x} u f(x ; u), \quad f(x ; u) \in \mathcal{P}\left(\mathbb{R}^{m}, \mathcal{M}_{k-1}\right) .
\end{aligned}
$$

4. The dual twistor operator $\mathcal{T}_{k}{ }^{*}: \mathcal{P}\left(\mathbb{R}^{m}, \mathcal{M}_{k}\right) \rightarrow \mathcal{P}\left(\mathbb{R}^{m}, u \mathcal{M}_{k-1}\right)$

$$
\begin{aligned}
\mathcal{T}_{k}{ }^{*} f(x ; u) & =\left(\frac{k+\Gamma_{u}}{2 k+m-2}\right) \partial_{x} f(x ; u) \\
& =\frac{1}{2}(1+H) \partial_{x} f(x ; u), \quad f(x ; u) \in \mathcal{P}\left(\mathbb{R}^{m}, \mathcal{M}_{k}\right)
\end{aligned}
$$

These operators are the entries of the following block form representation of the Dirac operator:

$$
\partial_{x}: \mathcal{P}\left(\mathbb{R}^{m}, \mathcal{H}_{k}\right) \rightarrow \mathcal{P}\left(\mathbb{R}^{m}, \mathcal{H}_{k}\right): \quad \partial_{x} \longleftrightarrow\left(\begin{array}{cc}
\mathcal{R}_{k} & \mathcal{T}_{k} \\
\mathcal{T}_{k}{ }^{*} & \mathcal{D}_{k-1}
\end{array}\right)
$$

Proposition 3. The operators $\mathcal{R}$ and $\mathcal{T}$ can be restricted to the subspaces $\mathcal{P}\left(\mathbb{R}^{m}, \mathcal{M}_{k}\right)$ and $\mathcal{P}\left(\mathbb{R}^{m}, u \mathcal{M}_{k}\right)$. Their restrictions are precisely the four fundamental operators

$$
\begin{array}{ll}
\left.\mathcal{R}\right|_{\mathcal{P}\left(\mathbb{R}^{m}, \mathcal{M}_{k}\right)}=\mathcal{R}_{k} & \left.\mathcal{R}\right|_{\mathcal{P}\left(\mathbb{R}^{m}, u \mathcal{M}_{k}\right)}=\mathcal{D}_{k-1} \\
\left.\mathcal{T}\right|_{\mathcal{P}\left(\mathbb{R}^{m}, \mathcal{M}_{k}\right)}=\mathcal{T}_{k} & \left.\mathcal{T}\right|_{\mathcal{P}\left(\mathbb{R}^{m}, u \mathcal{M}_{k}\right)}=\mathcal{T}_{k}{ }^{*} .
\end{array}
$$

Proof. This follows immediately from the $H$-form of the four operators.

Since $\mathcal{R}$ and $\mathcal{T}$ commute with the even part $\mathfrak{s l}(2) \cong\left\{\Delta_{u},|u|^{2}, \mathbb{E}_{u}+\frac{m}{2}\right\}$ of $\mathfrak{o s p}(1 \mid 2)$, it is enough to consider their action on the space of polynomials $\mathcal{P}\left(\mathbb{R}^{m}, \mathcal{H}\right)$ taking values in the $\mathfrak{s l}(2)$-lowest weight module $\mathcal{H}\left(\mathbb{R}^{m}, V\right)$, which decomposes as $\mathcal{M}\left(\mathbb{R}^{m}, V\right) \oplus u \mathcal{M}\left(\mathbb{R}^{m}, V\right)$ :

$$
\begin{array}{ll}
\left.\mathcal{R}\right|_{\mathcal{P}\left(\mathbb{R}^{m}, \mathcal{M}\right)}=\oplus_{k \geq 0} \mathcal{R}_{k} & \left.\mathcal{R}\right|_{\mathcal{P}\left(\mathbb{R}^{m}, u \mathcal{M}\right)}=\oplus_{k \geq 0} \mathcal{D}_{k} \\
\left.\mathcal{T}\right|_{\mathcal{P}\left(\mathbb{R}^{m}, \mathcal{M}\right)}=\oplus_{k \geq 0} \mathcal{T}_{k} & \left.\mathcal{T}\right|_{\mathcal{P}\left(\mathbb{R}^{m}, u \mathcal{M}\right)}=\oplus_{k \geq 0} \mathcal{T}_{k}{ }^{*} .
\end{array}
$$

\section{The total rescaled Rarita-Schwinger and twistor operator}

Due to the appearance of the integral operator $P_{1}^{-1}$ in the definition of the total RS- and twistor operator (see proposition 2), these operators lack the property of being differential operators on $\mathcal{P}\left(\mathbb{R}^{2 \times m}, V\right)$. This motivates us to rescale them in the following way.

Definition 4. The total rescaled RS and twistor operator on $\mathcal{P}\left(\mathbb{R}^{2 \times m}, V\right)$ are defined as

$$
\begin{aligned}
\mathcal{R}_{s} & :=P_{1} \mathcal{R}=\frac{1}{2}\left\{P_{1}, \partial_{x}\right\}=\frac{1}{2}\left\{m-2-2 \Gamma_{u}, \partial_{x}\right\} \\
\mathcal{T}_{s} & :=P_{1} \mathcal{T}=\frac{1}{2}\left[P_{1}, \partial_{x}\right]=\frac{1}{2}\left[m-2-2 \Gamma_{u}, \partial_{x}\right] .
\end{aligned}
$$


Due to this rescaling, $\mathcal{R}_{s}$ and $\mathcal{T}_{s}$ are differential operators which can be expressed in the algebra generated by $u, \partial_{u}$ and $\partial_{x}$. Although the operator $\mathcal{R}_{s}$ is no longer realized as the action of $\partial_{x}$ followed by a projection, there is a good reason to consider this rescaled operator. Recall that the unscaled $\mathcal{R}$ is already $\mathfrak{s l}(2)$-invariant. It turns out that the rescaled operator $\mathcal{R}_{s}$ has enhanced invariance properties. First of all we need the following:

Lemma 1. Let $a \in \mathbb{C}_{m}^{1}$ be an arbitrary vector. The operator defined by the anti-commutator

$$
\left\{a, P_{1}\right\}: \mathcal{P}\left(\mathbb{R}^{m}, V\right) \rightarrow \mathcal{P}\left(\mathbb{R}^{m}, V\right): f(u) \mapsto\left\{a, P_{1}\right\} f(u)
$$

commutes with the generators $u, \partial_{u}$ of the Lie superalgebra osp $(1 \mid 2)$.

Proof. It is sufficient to prove the statement for a fixed (unit) vector $e_{1} \in \mathbb{R}^{m}$. Consider the splitting $\mathbb{R}^{m}=\mathbb{R} e_{1} \oplus e_{1}^{\perp} \cong \mathbb{R} e_{1} \oplus \mathbb{R}^{m-1}$. Then $u=e_{1} u_{1}+\underline{u}$ and $\partial_{u}=e_{1} \partial_{u_{1}}+\partial_{\underline{u}}$. Using the definition of $\Gamma_{u}$ (on $\mathbb{R}^{m}$ ) and denoting the Gamma-operator on $\mathbb{R}^{m-1}$ by means of $\Gamma_{u}^{\prime}=-\sum_{1<i<j} e_{i j} L_{i j}$ we obtain:

$$
\begin{aligned}
\left\{e_{1}, P_{1}\right\} & =\left\{e_{1}, \frac{m-2}{2}-\Gamma_{u}\right\}=(m-2) e_{1}+\left\{e_{1}, \sum_{1<i} e_{1 i} L_{1 i}-\Gamma_{u}^{\prime}\right\} \\
& =(m-2) e_{1}-2 e_{1} \Gamma_{u}^{\prime}=2 e_{1} S c_{1}^{\prime},
\end{aligned}
$$

where $S c_{1}^{\prime}$ denotes the Scasimir operator on $\mathbb{R}^{m-1}$. In this way $S c_{1}^{\prime}$ can be regarded as the Scasimir of the $\mathfrak{o s p}(1 \mid 2)$-Lie superalgebra generated by $\underline{u}, \partial_{\underline{u}}$ and therefore anti-commutes with $\underline{u}, \partial_{\underline{u}}$. Clearly $e_{1} u_{1}$ commutes with both $e_{1}$ and $S c_{1}^{\prime}$ while $\underline{u}$ anti-commutes with both $e_{1}$ and $S c_{1}^{\prime}$. The same is true for $\partial_{u}$, from which it follows that $\left\{e_{1}, P_{1}\right\}$ commutes with the generators $u$ and $\partial_{u}$ of $\mathfrak{o s p}(1 \mid 2)$.

Lemma 2. Let $B$ and $P$ be operators such that $P^{2}$ commutes with $B$, then $\{P, B\}$ commutes with $P$ while $[P, B]$ anti-commutes with $P$. If moreover $B^{2}$ commutes with $P$, then also $\{P, B\}$ commutes with $[P, B]$.

Proof. Follows from easy calculations.

This leads to the following conclusions:

\section{Proposition 4.}

1. The rescaled operator $\mathcal{R}_{s}$ commutes with the generators $u, \partial_{u}$ of $\mathfrak{o s p}(1 \mid 2)$.

2. The rescaled operator $\mathcal{T}_{s}$ only commutes with the even part $\mathfrak{s l}(2)$ of $\mathfrak{o s p}(1 \mid 2)$.

3. We have that $\left[\mathcal{R}_{s}, \mathcal{T}_{s}\right]=\left[\mathcal{R}_{s}, P_{1}\right]=\left\{\mathcal{T}_{s}, P_{1}\right\}=0$.

Proof. Take $a$ to be the Dirac operator $\partial_{x}$ (which behaves like a vector) in lemma 1 . This proves the enhanced invariance property for $\mathcal{R}_{s}$. The invariance of $\mathcal{T}_{s}$ follows immediately from proposition 2. If we take $B=\partial_{x}$ and $P=P_{1}$, the conditions of lemma 2 are satisfied. This implies the third statement.

The operators $\mathcal{R}_{s}$ and $\mathcal{T}_{s}$ are still considered to be examples of even (resp. odd) operators. This follows from the (anti-)commutation relations with respect to $P_{1}$ : an operator (anti-)commuting with $P_{1}$ is even (odd). This notion of even and odd is exactly the same as the grading induced by the Hilbert operator $H$. Consider then for fixed $x$ the decomposition of polynomials $f(x ; u)$ under the Howe dual pair $\operatorname{Spin}(m) \times \mathfrak{o s p}(1 \mid 2)$ acting on the $u$-variable:

$$
\mathcal{P}\left(\mathbb{R}^{m}, V\right)=\bigoplus_{k \geq 0} \mathbb{W}_{k}^{\infty} \otimes \mathcal{M}_{k}\left(\mathbb{R}^{m}, V\right)
$$


D. Eelbode and P. Van Lancker

Because of the extra $\mathfrak{o s p}(1 \mid 2)$-invariance, $\mathcal{R}_{s}$ commutes with the first factor in each summand and it can thus be regarded as a direct sum of operators $\mathcal{P}\left(\mathbb{R}^{m}, \mathbb{W}_{k}^{\infty} \otimes \mathcal{M}_{k}\right) \rightarrow \mathcal{P}\left(\mathbb{R}^{m}, \mathbb{W}_{k}^{\infty} \otimes \mathcal{M}_{k}\right)$, for all $k \in \mathbb{N}$. It is therefore sufficient to consider its action on $\mathcal{P}\left(\mathbb{R}^{m}, \mathcal{M}_{k}\right)$ :

$$
\left.\mathcal{R}_{s}\right|_{\mathcal{P}\left(\mathbb{R}^{m}, \mathcal{M}_{k}\right)}=(2 k+m-2) \mathcal{R}_{k},
$$

which yields a $k$-dependent scalar multiple of the Rarita-Schwinger operator $\mathcal{R}_{k}$ introduced in $[4,5,16]$. It can be proved that $\mathcal{R}_{k}$ is the unique (up to a scalar multiple) conformally invariant first order constant coefficient differential operator defined on $\mathcal{P}\left(\mathbb{R}^{m}, \mathcal{M}_{k}\right)$, see e.g. [11]. As we will point out in the next section, the operator $\mathcal{R}_{s}$ inherits the conformal invariance properties of the Rarita-Schwinger operators $\mathcal{R}_{k}$, see also [9]. Our definition of the $\mathcal{R}_{s}$-operator thus incorporates all the formerly defined operators $\mathcal{R}_{k}$ (where precisely the freedom of choice of a scalar multiple depending on $k$ is exploited to scale each of them in a particular way) into one single operator. Using $\mathcal{R}_{s}$, it does not matter how the space $\mathcal{M}_{k}\left(\mathbb{R}^{m}, V\right)$, as a model of a $\operatorname{Spin}(m)$-representation with highest weight $\left(k+\frac{1}{2}, \frac{1}{2}, \cdots, \frac{1}{2}, \pm \frac{1}{2}\right)$, is embedded into the full space $\mathcal{P}\left(\mathbb{R}^{m}, V\right)$ of polynomials in $u \in \mathbb{R}^{m}$. Moreover, as explained in [4], the structure of the kernel of $\mathcal{R}_{k}$ as $\operatorname{Spin}(m)$-representation can be determined inductively in terms of the kernels of $\mathcal{R}_{j}, j=0, \ldots, k-1$. This, together with their simple appearance, is an extra motivation for considering the rescaled operators $\mathcal{R}_{s}$ and $\mathcal{T}_{s}$.

\section{Conformal Invariance}

In this section, we investigate how the rescaled Rarita-Schwinger operator $\mathcal{R}_{s}$ transforms under the conformal group $\operatorname{Spin}(m+1,1)$. This group is generated by rotations, translations, dilations and the inversion. In the framework of Clifford analysis, the group $\operatorname{Spin}(m+1,1)$ is usually described in terms of Vahlen matrices (see e.g. the work [1] by Ahlfors). The behaviour of the Rarita-Schwinger operator $\mathcal{R}_{k}$ (acting on $\mathcal{M}_{k}$-valued functions) under the action of the conformal group has also been elaborated in the paper [9]. Define the following representations of the subsequent groups acting on an arbitrary function $f(x ; u)$ :

1. $(L(s) f)(x ; u):=s f(\bar{s} x s ; \bar{s} u s), s \in \operatorname{Spin}(m)$ (rotations)

2. $(T(a) f)(x ; u):=f(x-a ; u), a \in \mathbb{R}^{m}$ (translations)

3. $(D(\lambda) f)(x ; u):=\lambda^{\frac{m-1}{2}} f(\lambda x ; u), \lambda \in \mathbb{R}$ (dilations) .

The transformation behaviour of $\mathcal{R}_{s}$ under these representations is clear. First of all, $\mathcal{R}_{s}$ commutes with the above defined $L$ - and $T$-representation: this follows at once from the fact that $\mathcal{R}_{s}$ can be expressed solely in terms of $u, \partial_{u}$ and $\partial_{x}$ (see definition 4) and each of these operators is $\operatorname{Spin}(m)$-invariants. Moreover $\mathcal{R}_{s} D(\lambda)=\lambda D(\lambda) \mathcal{R}_{s}$. So we only need to know how the inversion acts on $f$. This turns out to be the most complicated action. We therefor fix the following notations and definitions: the (monogenic) inversion operator $\mathcal{I}_{M}$ acts on functions $f(x)$ by

$$
\left(\mathcal{I}_{M} f\right)(x):=\frac{x}{|x|^{m}} f\left(\frac{x}{|x|^{2}}\right) .
$$

The inversion operator $\mathcal{I}_{M}$ maps monogenic functions to monogenic functions. This property follows easily from the basic operator identity:

$$
\mathcal{I}_{M} \partial_{x} \mathcal{I}_{M}=|x|^{2} \partial_{x}
$$

The $H$-action of $\omega=\frac{x}{|x|}$ on the dummy variable $u$ defines a reflection of $u$ in the hyperplane orthogonal to $\omega$. Its image can be expressed as

$$
h(\omega) u:=\frac{x u x}{|x|^{2}}=\omega u \omega=\omega\left(\langle\omega, u\rangle \omega+u^{\perp}\right) \omega=u-2\langle\omega, u\rangle \omega
$$


and the induced action on functions $g(u)$ is then given by $(h(\omega) g)(u)=g(\omega u \omega)$. The inversion operator for the Rarita-Schwinger operator $\mathcal{R}_{k}$ introduced in $[4,5,9]$ leads to the following definition, valid on the full space $\mathcal{P}\left(\mathbb{R}^{2 \times m}\right)$ :

Definition 5. The inversion operator $\mathcal{I}$ acting on functions $f(x ; u) \in \mathcal{P}\left(\mathbb{R}^{2 \times m}\right)$ is the operator $\mathcal{I}:=\mathcal{I}_{M} \otimes h(\omega)$, i.e.

$$
(\mathcal{I} f)(x ; u):=\frac{x}{|x|^{m}} f\left(\frac{x}{|x|^{2}} ; \omega u \omega\right)=|x|^{-m+1} \omega f\left(\frac{x}{|x|^{2}} ; \omega u \omega\right) .
$$

Note that this definition is equivalent with the more natural definition $\mathcal{I}:=\mathcal{I}_{-m+1} \otimes L(\omega)$ where $L$ is the usual representation of $\operatorname{Spin}(m)$ and

$$
\left(\mathcal{I}_{-m+1} f\right)(x)=|x|^{-m+1} f\left(\frac{x}{|x|^{2}}\right) .
$$

For computational reasons we will use the first definition of $\mathcal{I}$.

Lemma 3. For arbitrary functions $g(u)$ we have:

$$
h(\omega) \partial_{x} h(\omega) g(u)=-\left[\Gamma_{u}, \frac{x}{|x|^{2}}\right] g(u) .
$$

Proof. Put $x=|x| \omega$ and $v=h(\omega) u=u-2\langle\omega, u\rangle \omega$. Then

$$
\frac{\partial v_{j}}{\partial x_{i}}=-2 \frac{\partial}{\partial x_{i}}\left(\frac{\langle x, u\rangle}{|x|^{2}} x_{j}\right)=-\frac{2}{|x|^{2}}\left(u_{i} x_{j}+\langle x, u\rangle \delta_{i j}-2\langle x, u\rangle \frac{x_{i} x_{j}}{|x|^{2}}\right) .
$$

As a result, we thus have that

$$
\begin{aligned}
\partial_{x} g(h(\omega) u) & =-\frac{2}{|x|^{2}} \sum_{i, j=1}^{m} e_{i}\left(u_{i} x_{j}+\langle x, u\rangle \delta_{i j}-2\langle x, u\rangle \frac{x_{i} x_{j}}{|x|^{2}}\right) \frac{\partial g}{\partial v_{j}}(v) \\
& =-\frac{2}{|x|^{2}}\left(u\left\langle x, \partial_{v}\right\rangle+\langle x, u\rangle \partial_{v}-2 \frac{x}{|x|^{2}}\langle x, u\rangle\left\langle x, \partial_{v}\right\rangle\right) g(v),
\end{aligned}
$$

where we have put $v=h(\omega) u$ in both expressions on the right hand side. In view of the fact that $\langle x, v\rangle=\langle x, u-2\langle\omega, u\rangle \omega\rangle=-\langle x, u\rangle$, we thus obtain:

$$
h(\omega) \partial_{x} h(\omega) g(u)=-\frac{2}{|x|^{2}}\left(\frac{x u x}{|x|^{2}}\left\langle x, \partial_{u}\right\rangle-\langle x, u\rangle \partial_{u}+2 \frac{x}{|x|^{2}}\langle x, u\rangle\left\langle x, \partial_{u}\right\rangle\right) g(u) .
$$

Putting $x=|x| \omega$, it is easily seen that this simplifies to

$$
h(\omega) \partial_{x} h(\omega) g(u)=-\frac{2}{|x|}\left(u\left\langle\omega, \partial_{u}\right\rangle-\langle\omega, u\rangle \partial_{u}\right) g(u) \text {. }
$$

On the other hand,

$$
\left[\Gamma_{u}, \omega\right]=-\left[u \partial_{u}, \omega\right]=\left(\omega u \partial_{u}-u \partial_{u} \omega\right)=2\left(u\left\langle\omega, \partial_{u}\right\rangle-\langle\omega, u\rangle \partial_{u}\right),
$$

which thus proves the statement.

Corollary 1. For functions depending on $(x ; u) \in \mathbb{R}^{2 \times m}$, we get:

$$
h(\omega) \partial_{x} h(\omega) f(x ; u)=\left(\partial_{x}-\frac{1}{|x|^{2}}\left[\Gamma_{u}, x\right]\right) f(x ; u) .
$$


We can now easily obtain the following result:

Lemma 4. On arbitrary functions $f(x ; u)$, one has the operator identity

$$
\mathcal{I} \partial_{x} \mathcal{I}=|x|^{2} \partial_{x}-\left[\Gamma_{u}, x\right]
$$

Proof. In view of the operator identity (6) and the previous corollary:

$$
\begin{aligned}
\mathcal{I} \partial_{x} \mathcal{I} & =\mathcal{I}_{M} h(\omega) \partial_{x} h(\omega) \mathcal{I}_{M} \\
& =\mathcal{I}_{M} \partial_{x} \mathcal{I}_{M}-\mathcal{I}_{M} \frac{1}{|x|^{2}}\left[\Gamma_{u}, x\right] \mathcal{I}_{M} \\
& =|x|^{2} \partial_{x}-|x| \omega\left[\Gamma_{u}, \omega\right] \omega \\
& =|x|^{2} \partial_{x}-\left[\Gamma_{u}, x\right] .
\end{aligned}
$$

Note that the second term at the right-hand side can be rewritten as $\left[\Gamma_{u}, x\right]=\left[x, P_{1}\right]$.

Lemma 5. The inversion operator $\mathcal{I}$ anti-commutes with the odd part of the algebra osp $(1 \mid 2)$ (in the variable $u$ ), from which it follows that $\left[P_{1}, \mathcal{I}\right]=0$.

Proof. This can be checked by direct computations.

Proposition 5. The operator $\mathcal{R}_{s}$ is invariant with respect to $\mathcal{I}$, i.e.

$$
\mathcal{I} \mathcal{R}_{s} \mathcal{I}=|x|^{2} \mathcal{R}_{s}
$$

when acting on arbitrary functions $f(x ; u)$.

Proof. By lemma 4 and 5 we have the operator identity

$$
P_{1} \mathcal{I} \partial_{x} \mathcal{I}=\mathcal{I} P_{1} \partial_{x} \mathcal{I}=|x|^{2} P_{1} \partial_{x}+P_{1}\left[x, P_{1}\right]
$$

On the other hand, one has that

$$
\mathcal{I} \partial_{x} \mathcal{I} P_{1}=\mathcal{I} \partial_{x} P_{1} \mathcal{I}=|x|^{2} \partial_{x} P_{1}+\left[x, P_{1}\right] P_{1}
$$

It then suffices to note that $P_{1}\left[x, P_{1}\right]+\left[x, P_{1}\right] P_{1}=\left[x, P_{1}^{2}\right]=0$, which follows from the fact that the operator $P_{1}^{2}$ is scalar (see expression (5)). Adding both identities then leads to the desired result.

Expression (7) shows that the inversion operator $\mathcal{I}$ (with a more complicated action) behaves in the same way with respect to the rescaled Rarita-Schwinger $\mathcal{R}_{s}$ as the operator $\mathcal{I}_{M}$ with respect to the Dirac operator $\partial_{x}$. In particular, $\mathcal{I}$ preserves the kernel of $\mathcal{R}_{s}$. This is the starting point to construct the fundamental solution of $\mathcal{R}_{s}$.

\section{$9 \quad$ Fundamental solutions}

The aim of this section is to construct a fundamental solution $E(x ; u, v)$ for the operator $\mathcal{R}_{s}$, acting on the total space $\mathcal{M}$ of monogenic polynomials in the variable $u$. To do so, we want to find a homogeneous solution, in distributional sense, to the equation $\mathcal{R}_{s} E(x ; u, v)=\delta(x) K(u, v)$, where the identity of the space $\operatorname{End}(\mathcal{M})$ is represented by the reproducing kernel $K(u, v)$. For the following result we refer to e.g. [8]: 
Definition 6. Let $u=|u| e_{u}, v=|v| e_{v}$ and $t=\left\langle e_{u}, e_{v}\right\rangle \in[-1,1]$. The reproducing kernel $K_{k}(u, v)$ of $\mathcal{M}_{k}\left(\mathbb{R}^{m}\right)$ is given by

$$
K_{k}(u, v)=|x|^{k}|u|^{k} K_{k}\left(e_{u}, e_{v}\right):=|x|^{k}|u|^{k}\left(C_{k}^{\frac{m}{2}}(t)+C_{k-1}^{\frac{m}{2}}(t) e_{u} e_{v}\right),
$$

where $C_{k}^{\frac{m}{2}}(t)$ is a Gegenbauer polynomial and:

$$
M_{k}\left(e_{v}\right)=\frac{1}{A_{m}} \int_{S^{m-1}} \overline{K_{k}\left(e_{u}, e_{v}\right)} M_{k}\left(e_{u}\right) d e_{u}, \quad M_{k} \in \mathcal{M}_{k}\left(\mathbb{R}^{m}\right) .
$$

Hereby $d e_{u}$ denotes the invariant measure on $S^{m-1}$ and $A_{m}$ the surface area of $S^{m-1}$.

We will now first focus on a fixed degree of homogeneity $k \in \mathbb{N}$, and switch to the total space $\mathcal{M}$ at the end of the calculations. The space $\mathcal{M}_{k}\left(\mathbb{R}^{m}, \mathbb{C}_{m}^{+}\right)$is a direct sum of equivalent irreducible $\operatorname{Spin}(m)$-representations (for the $L$-action), each of them being generated by a highest weight vector $P_{k}(u, \tau):=\langle u, \tau\rangle^{k} \tau \bar{\tau}$ for a suitable choice of null vector $\tau,\left(\tau^{2}=0\right)$. Therefore $K_{k}(u, v)$ can be expressed as a linear combination of $L(s) P_{k}(u, \tau)$ where $\tau$ varies in the set of chosen null vectors. In the sequel we will derive various identities involving $\operatorname{Spin}(m)$-invariant operators acting on $P_{k}(u, \tau)$. The afore-mentioned remark thus implies that such identies remain valid when acting on $\mathcal{M}_{k}\left(\mathbb{R}^{m}, \mathbb{C}_{m}^{+}\right)$, or in particular on $K_{k}(u, v)$. Therefore we now focus on the polynomial $P_{k}(u, \tau)$ (which is easier from computational point of view).

Lemma 6. For all $k \in \mathbb{N}$, the plane wave $P_{k}(u, \tau)$ satisfies:

(i) The function $u \mapsto P_{k}(x u x, \tau)$ is harmonic in $u$

(ii) The function $u \mapsto x P_{k}(x u x, \tau)$ is monogenic in $u$

(iii) $\partial_{x}\left(x P_{k}(x u x, \tau)\right)=-(m+2 k) P_{k}(x u x, \tau)-2 k u x P_{k-1}(x u x, \tau)\langle x, \tau\rangle$.

(iv) For $\alpha \in \mathbb{C}$, the homogeneous function defined by

$$
F_{\alpha, k}(x ; u, \tau):=x|x|^{\alpha-2 k} P_{k}(x u x, \tau) \in C^{\infty}\left(\mathbb{R}_{0}^{m}, \mathcal{M}_{k}\right)
$$

satisfies $\mathcal{R}_{k} F_{\alpha, k}(x ; u, \tau)=-(\alpha+m)|x|^{\alpha-2 k} \pi_{k}\left[P_{k}(x u x, \tau)\right]$, where $\pi_{k}$ is defined as the projection $\pi_{k}: \mathcal{H}_{k}\left(\mathbb{R}^{m}, V\right) \rightarrow \mathcal{M}_{k}\left(\mathbb{R}^{m}, V\right)$.

Proof. For an arbitrary homogeneous polynomial $P_{k}(u)$ of degree $k$ :

$$
P_{k}(x u x)=|x|^{2 k}\left(h(\omega) P_{k}\right)(u) \quad \text { and } \quad x P_{k}(x u x)=|x|^{2 k+1}\left(L(\omega) P_{k}\right)(u) .
$$

The actions $h(\omega)$ and $L(\omega)$ preserve harmonic, resp. monogenic polynomials in $u$. This implies (i) and (ii). Property (iii) follows from straightforward calculations, invoking the definition

$$
P_{k}(x u x)=\left(|x|^{2}\langle u, \tau\rangle-2\langle x, \tau\rangle\langle x, u\rangle\right)^{k} \tau \bar{\tau} .
$$

By (iii), we get:

$$
\begin{aligned}
\partial_{x} F_{\alpha, k}(x ; u, \tau) & =(\alpha-2 k) x|x|^{\alpha-2 k-2} x P_{k}(x u x, \tau) \\
& +|x|^{\alpha-2 k}\left(-(m+2 k) P_{k}(x u x, \tau)-2 k u x P_{k-1}(x u x, \tau)\langle x, \tau\rangle\right) \\
& =-(\alpha+m) x F_{\alpha-2, k}(x ; u, \tau)-2 k u F_{\alpha-2, k-1}(x ; u, \tau)\langle x, \tau\rangle .
\end{aligned}
$$

The left hand side defines a spherical harmonic of order $k$ in $u$. Applying the projection operator $\pi_{k}$ to this expression yields $(i v)$. 
Lemma 7. The map $\alpha \mapsto F_{\alpha, k}(x ; u, \tau)$ can be extended to a holomorphic map $\mathbb{C} \backslash\{-(m+2 p+2)$ : $p \in \mathbb{N}\} \rightarrow \mathcal{D}^{\prime}\left(\mathbb{R}^{m}, \mathcal{M}_{k}\right)$ having simple poles in the excluded points. The homogeneous distribution $F_{\alpha, k}(x ; u, \tau)$ satisfies:

$$
\mathcal{R}_{k} F_{-m, k}(x ; u, \tau)=-\frac{\pi^{\frac{m}{2}} 2^{1-2 k}}{\Gamma\left(k+\frac{m}{2}\right) k !}\left(\pi_{k}\left[\Delta_{x}^{k} P_{k}(x u x, \tau)\right]\right) \delta(x) .
$$

Proof. For $\Re(\alpha)>-(m+1)$, the function $x \mapsto F_{\alpha, k}(x ; u, \tau)$ belongs to the function space $L_{1}^{\text {loc }}\left(\mathbb{R}^{m}, \mathcal{M}_{k}\right)$, which means that it defines a distribution in $\mathcal{D}^{\prime}\left(\mathbb{R}^{m}, \mathcal{M}_{k}\right)$ for these values for $\alpha$. This distribution depends holomorphically on $\alpha$ and can be extended to a meromorphic function on $\mathbb{C}$. Indeed, $F_{\alpha, k}(x ; u, \tau)$ can be seen as the product of the power $|x|^{\alpha-2 k}$ and a polynomial in $x \in \mathbb{R}^{m}$. Since the former defines a distribution on $\mathcal{D}^{\prime}\left(\mathbb{R}^{m}\right)$ or $\mathcal{S}^{\prime}\left(\mathbb{R}^{m}\right)$, it is clear how to define $F_{\alpha, k}(x ; u, \tau)$ as a distribution. Putting $\alpha=-m$, we obtain by $(i v)$ of the previous lemma a function $F_{-m, k}(x ; u)$ satisfying $\mathcal{R}_{k} F_{-m, k}(x ; u)=0$ in $\mathbb{R}_{0}^{m}$ and having a singularity of degree $-(m-1)$ at the origin. In fact, this also follows from the relation

$$
F_{-m, k}(x ; u, \tau):=x|x|^{-m-2 k} P_{k}(x u x, \tau)=\left(\mathcal{I} P_{k}\right)(x ; u)
$$

and the conformal invariance property (5). To compute $\mathcal{R}_{k} F_{-m, k}(x ; u, \tau)$ in distributional sense we will use the so-called Riesz potentials. For $\Re(\lambda)>-m$, the generalized powers $|x|^{\lambda}$ define an element of $\mathcal{D}^{\prime}\left(\mathbb{R}^{m}\right)$ satisfying $\Delta_{x}|x|^{\lambda}=\lambda(\lambda+m-2)|x|^{\lambda-2}$ in distributional sense. This leads to the definition of the Riesz potentials $I_{\lambda} \in \mathcal{S}^{\prime}\left(\mathbb{R}^{m}\right)$ (see e.g. the work [14] of Gel'fand and Shilov). We will summarize here the results which are of interest to us:

$$
I_{\lambda}:=\frac{\Gamma\left(-\frac{\lambda}{2}\right)}{\pi^{\frac{m}{2}} 2^{\lambda} \Gamma\left(\frac{\lambda+m}{2}\right)}|x|^{\lambda} .
$$

For $\Re(\lambda)>-m$, we have that $\Delta_{x} I_{\lambda}=-I_{\lambda-2}$ in distributional sense. This identity can be used to obtain an entire function $\lambda \mapsto I_{\lambda}$. Indeed, for $\Re(\lambda)>-(m+2 p)$, one has $I_{\lambda}=(-1)^{p} \Delta_{x}^{p} I_{\lambda+2 p}$. Therefore, one can shift the domain for $\lambda$ to $\Re(\lambda)>-(m+2 p)$. In the points $\lambda=-m-2 p$ (with $p \in \mathbb{N}$ ), one obtains the homogeneous distributions

$$
I_{-m-2 p}=(-1)^{p} 2^{m+1} \Delta_{x}^{p} \delta(x)
$$

with support in the origin. In terms of the original distribution $|x|^{\lambda}$, one obtains a holomorphic extension to $\mathbb{C} \backslash\{-m-2 p: p \in \mathbb{N}\}$. The poles in the excluded points are simple, and the residues are given by

$$
\operatorname{Res}\left[|x|^{\lambda}, \lambda=-m-2 p\right]=\frac{\pi^{\frac{m}{2}} 2^{1-2 p}}{\Gamma\left(p+\frac{m}{2}\right) p !} \Delta_{x}^{p} \delta(x) .
$$

Returning to $\alpha \mapsto F_{\alpha, k}(x ; u, \tau)=|x|^{\alpha-2 k} x P_{k}(x u x, \tau)$, it is clear that this function can initially be extended to $\mathbb{C} \backslash\{-m-2 k-2 p: p \in \mathbb{N}\}$. The poles of the form $-m,-m+2, \cdots$ are removable (follows from calculating the residue), which means that the function is holomorphic in $\mathbb{C} \backslash\{-(m+2 p+2): p \in \mathbb{N}\}$. We thus have:

$$
\begin{aligned}
\mathcal{R}_{k} F_{-m, k}(x ; u, \tau) & =-\operatorname{Res}\left[|x|^{\alpha-2 k}, \alpha=-m\right] \pi_{k}\left[P_{k}(x u x, \tau)\right] \\
& =-\frac{\pi^{\frac{m}{2}} 2^{1-2 k}}{\Gamma\left(k+\frac{m}{2}\right) k !} \Delta_{x}^{k} \delta(x) \pi_{k}\left[P_{k}(x u x, \tau)\right] \\
& =-\frac{\pi^{\frac{m}{2}} 2^{1-2 k}}{\Gamma\left(k+\frac{m}{2}\right) k !}\left(\pi_{k}\left[\Delta_{x}^{k} P_{k}(x u x, \tau)\right]\right) \delta(x) .
\end{aligned}
$$

This proves the lemma. 
In order to determine the projection $\pi_{k}\left[\Delta_{x}^{k} P_{k}(x u x, \tau)\right]$ in the last line, we proceed as follows. The first step is to determine $\Delta_{x}^{k} P_{k}(x u x, \tau)$. As we will see shortly, the main property needed here is that $P_{k}(u, \tau)$ is harmonic in $u$. Therefore we will focus our attention on $\Delta_{x}^{k} H_{k}(x u x)$, with $H_{k}(u) \in \mathcal{H}_{k}\left(\mathbb{R}^{m}\right)$. The space $\mathcal{H}_{k}\left(\mathbb{R}^{m}\right)$ is irreducible under the $h$-action of the group $\operatorname{Spin}(m)$. Hence it is the linear span of $h(s) w_{k}(u, \tau)$, with $w_{k}(u, \tau):=\langle u, \tau\rangle^{k}$ a highest weight vector for $\mathcal{H}_{k}\left(\mathbb{R}^{m}\right)$. We then define the map

$$
h(x): \mathcal{H}_{k} \rightarrow \mathcal{P}_{2 k} \otimes \mathcal{H}_{k}: H_{k}(u) \mapsto H_{k}(x u x) .
$$

This map is the unique $2 k$-homogeneous polynomial extension of the map $\omega \mapsto h(\omega) H_{k}(u)$, with $\omega \in S^{m-1}$. An important feature of the map $h(x)$ is that its composition with $\Delta_{x}^{k}$ yields a constant multiple of the identity on $\mathcal{H}_{k}\left(\mathbb{R}^{m}\right)$ :

Lemma 8. Let $H_{k}(u) \in \mathcal{H}_{k}\left(\mathbb{R}^{m}\right)$ be arbitrary. Then the following holds:

$$
\Delta_{x}^{k}\left(h(x) H_{k}(u)\right)=\Delta_{x}^{k} H_{k}(x u x)=2^{2 k} k ! \frac{\Gamma\left(k+\frac{m-2}{2}\right)}{\Gamma\left(\frac{m}{2}\right)} H_{k}(u) .
$$

Proof. Since $H_{k}(x u x) \in \mathcal{P}_{2 k} \otimes \mathcal{H}_{k}$, we have that $\Delta_{x}^{k} H_{k}(x u x) \in \mathcal{H}_{k}$ and is independent of $x$. For any $s \in \operatorname{Spin}(m)$, we then consider the $h(s)$-action on the variable $u$ and the action $h_{2}(s):=(h \otimes h)(s)$ on $(x ; u)$. Both actions clearly commute with the operator $\Delta_{x}$, and for $H_{k} \in \mathcal{H}_{k}\left(\mathbb{R}^{m}\right)$ we have:

$$
\left(h(x) h(s) H_{k}\right)(u)=H_{k}(\bar{s} x u x s)=\left(h_{2}(s) h(x) H_{k}\right)(u) .
$$

As a result, the composed map $\Delta_{x}^{k} h(x): \mathcal{H}_{k} \rightarrow \mathcal{H}_{k}$ commutes with the $h$-action of $\operatorname{Spin}(m)$. Therefore, Schur's lemma implies the existence of a constant $C_{k}$ such that for all $u \in \mathbb{R}^{m}$ the following holds:

$$
\Delta_{x}^{k} H_{k}(x u x)=C_{k} H_{k}(u) .
$$

In order to fix this constant, it suffices to make a particular choice: we take $H_{k}(u)=w_{k}(u, \tau)$, with

$$
u=\frac{\sqrt{2}}{2}\left(e_{1}-i e_{2}\right) \text { and } \tau=\frac{\sqrt{2}}{2}\left(e_{1}+i e_{2}\right),
$$

satisfying $\langle u, \tau\rangle=1$. Introduce $z:=x-\left(x_{1} e_{1}+x_{2} e_{2}\right) \in \mathbb{R}^{m-2}$, then:

$$
\langle x u x, \tau\rangle=|x|^{2}\langle u, \tau\rangle-2\langle x, \tau\rangle\langle x, u\rangle=|x|^{2}-\left(x_{1}^{2}+x_{2}^{2}\right)=|z|^{2} .
$$

Denoting the Laplace operator on $\mathbb{R}^{m-2}$ by means of $\Delta_{z}$, this yields:

$$
\Delta_{x}\langle x u x, \tau\rangle^{k}=\Delta_{x}^{k}|z|^{2 k}=\Delta_{z}^{k}|z|^{2 k} .
$$

Recalling (1) and using standard calculations in the algebra $\mathcal{U}(\mathfrak{s l}(2))$, or invoking the polar decomposition for the Laplace operator on $\mathbb{R}^{m}$, it is easily verified that

$$
\Delta_{x}|x|^{2 k}=2^{2 k} k ! \frac{\Gamma\left(k+\frac{m}{2}\right)}{\Gamma\left(\frac{m}{2}\right)} .
$$

Therefore, with the choices for $u$ and $\tau$ from above, we get:

$$
\Delta_{x}^{k} w_{k}(x u x, \tau)=\Delta_{z}^{k}|z|^{2 k}=2^{2 k} k ! \frac{\Gamma\left(k+\frac{m-2}{2}\right)}{\Gamma\left(\frac{m}{2}\right)}=C_{k} w_{k}(u, \tau)=C_{k} .
$$

Since $\Delta_{x}^{k} h(x)$ commutes with the $h$-action on $\mathcal{H}_{k}$, the result $(9)$ follows. 
We can now finally determine the fundamental solution $E_{k}(x ; u, v)$ of the Rarita-Schwinger operator $\mathcal{R}_{k}$. The expression for $E_{k}(x ; u, v)$ we give here was first obtained in [4]. For an alternative proof we also refer to [9]. In both cases, the proof is based on the Cauchy integral formula for $\mathcal{R}_{k}$.

Proposition 6. Put $x=|x| \omega$. The fundamental solution of $\mathcal{R}_{k}$ is given by

$$
E_{k}(x ; u, v):=-\frac{1}{A_{m}} \frac{2 k+m-2}{m-2} \frac{x}{|x|^{m}} K_{k}(\omega u \omega ; v) \in C^{\infty}\left(\mathbb{R}_{0}^{m}, \operatorname{End}\left(\mathcal{M}_{k}\right)\right)
$$

and satisfies $\mathcal{R}_{k} E_{k}(x ; u, v)=\delta(x) K_{k}(u ; v)$.

Proof. In view of lemma 8 and 9, we thus have the following normalization:

$$
\mathcal{R}_{k} F_{-m, k}(x ; u, \tau)=-\frac{4 \pi^{\frac{m}{2}}}{(2 k+m-2) \Gamma\left(\frac{m-2}{2}\right)} \delta(x) P_{k}(u, \tau) .
$$

Introducing the surface area $A_{m}$ of $S^{m-1}$, given by $A_{m}=\frac{2 \pi^{\frac{m}{2}}}{\Gamma\left(\frac{m}{2}\right)}$, we are thus lead to the following conclusion:

$$
(2 k+m-2) \mathcal{R}_{k}\left(-\frac{1}{(m-2) A_{m}} F_{-m, k}(x ; u, \tau)\right)=\delta(x) P_{k}(u, \tau) .
$$

The statement then follows by replacing $P_{k}(u, \tau)$ by $K_{k}(u, v)$ as explained before.

We now give some examples where the rescaled operator $\mathcal{R}_{s}$ appears in a natural way as an operator acting on functions $f \in C^{\infty}(\Omega, U), \Omega$ being an open subset of $\mathbb{R}^{m}$. Here, we take $U$ to be an (infinite dimensional) $L^{2}$-space obtained by taking the closure of $\mathcal{M}\left(\mathbb{R}^{m}, V\right)$ or $\mathcal{P}\left(\mathbb{R}^{m}, V\right)$ with respect to a chosen inner product.

\section{Example 1}

The first example deals with $\mathcal{R}_{s}$ acting on functions $f(x)$ taking values in a Hardy space of monogenic functions inside the unit ball $B_{m}(1)$ of $\mathbb{R}^{m}$, see e.g. $[13,15]$. Define

$$
H^{2}\left(\mathcal{M}\left(B_{m}\right)\right):=\left\{f(u) \in \mathcal{M}\left(B_{m}(1)\right): \sup _{0<|u|<1} \int_{S^{m-1}}|f(u)|^{2} d e_{u}<+\infty\right\} .
$$

By taking boundary values in $L^{2}$-sense, this Hardy space is isomorphic with

$$
H^{2}\left(S^{m-1}\right):=\left\{\lim _{r \rightarrow 1} f(r \omega): f(u) \in H^{2}\left(\mathcal{M}\left(B_{m}\right)\right)\right\}
$$

which admits the $L^{2}$-decomposition

$$
H^{2}\left(S^{m-1}\right)=\bigoplus_{k=0}^{\infty} \mathcal{M}_{k}\left(S^{m-1}\right)
$$

This is the subspace of $L^{2}\left(S^{m-1}, V\right)$ on which the Hilbert transform $H_{S}$ acts like the identity. We may now let $\mathcal{R}_{s}$ act on $C^{\infty}\left(\Omega, H^{2}\left(S^{m-1}\right)\right)$ and look for the corresponding fundamental solution. Consider the expansion in $L^{2}\left(S^{m-1}, \mathbb{C}_{m}\right)$ (see e.g. [8]):

$$
S\left(e_{u}, e_{v}\right):=\frac{1+e_{u} e_{v}}{\left|e_{u}-e_{v}\right|^{m}}=\sum_{k=0}^{\infty} K_{k}\left(e_{u}, e_{v}\right)
$$


The function on the left-hand side is the reproducing kernel of $H^{2}\left(S^{m-1}\right)$ which may be identified with the boundary value on the unit sphere $S^{m-1}$ of the Szegö kernel for the Hardy space $H^{2}\left(\mathcal{M}\left(B_{m}\right)\right)$. In view of $(10)$ in proposition 6 , we obtain, replacing $(2 k+m-2) \mathcal{R}_{k}$ by $\mathcal{R}_{s}$ :

$$
\mathcal{R}_{s}\left(-\frac{1}{(m-2) A_{m}} \frac{x}{|x|^{m}} K_{k}\left(\omega e_{u} \omega, e_{v}\right)\right)=\delta(x) K_{k}\left(e_{u}, e_{v}\right) .
$$

Taking the sum over all $k \in \mathbb{N}$ in $L^{2}$-sense yields:

$$
\mathcal{R}_{s}\left(-\frac{1}{(m-2) A_{m}} \frac{x}{|x|^{m}} S\left(\omega e_{u} \omega, e_{v}\right)\right)=\delta(x) S\left(e_{u}, e_{v}\right) .
$$

This leads to the following

Proposition 7. Let $S\left(e_{u}, e_{v}\right)$ be the reproducing kernel of the Hardy space $H^{2}\left(S^{m-1}\right)$. The kernel $E\left(x ; e_{u}, e_{v}\right)$ defined by

$$
E\left(x ; e_{u}, e_{v}\right):=-\frac{1}{(m-2) A_{m}} \frac{x}{|x|^{m}} S\left(\omega e_{u} \omega, e_{v}\right) \in C^{\infty}\left(\mathbb{R}_{0}^{m}, \operatorname{End}\left(H^{2}\left(S^{m-1}\right)\right)\right)
$$

satisfies $\mathcal{R}_{s} E\left(x ; e_{u}, e_{v}\right)=\delta(x) S\left(e_{u}, e_{v}\right)$ and one has in $L^{2}$-sense (with respect to $e_{u} \in S^{m-1}$ and with $e_{v} \in S^{m-1}$ fixed) :

$$
E\left(x ; e_{u}, e_{v}\right)=\sum_{k=0}^{\infty} \frac{E_{k}\left(x ; e_{u}, e_{v}\right)}{2 k+m-2} .
$$

\section{Example 2}

As the operator $\mathcal{R}_{s}$ commutes with the algebra $\mathfrak{o s p}(1 \mid 2)$, we can now also derive a fundamental solution for the operator $\mathcal{R}_{s}$ acting on the full space of polynomials in the vector variable $u \in \mathbb{R}^{m}$. To do so, we need the following:

Definition 7. Let $P, Q \in \mathcal{P}\left(\mathbb{R}^{m}, V\right)$. The Fischer inner product of $P$ and $Q$ is the positive definite Hermitean inner product defined as

$$
\langle P, Q\rangle:=\left.\left[P^{\dagger}\left(\partial_{u}\right) Q(u)\right]_{0}\right|_{u=0},
$$

where the dagger $\dagger$ denotes the Hermitean conjugation, which is the tensor product of the Clifford conjugation and the classical complex conjugation.

Let $\mathcal{F}$ be the closure of $\mathcal{P}\left(\mathbb{R}^{m}, V\right)$ with respect to the Fischer inner product. The reproducing kernel of $\mathcal{F}$ is the exponential function $e^{\langle u, v\rangle}$ because

$$
P(u)=\left\langle e^{\langle u, v\rangle}, Q(v)\right\rangle .
$$

We may now consider the action of $\mathcal{R}_{s}$ on $C^{\infty}(\Omega, \mathcal{F})$. We thus obtain:

Proposition 8. The fundamental solution $F(x ; u, v)$ of $\mathcal{R}_{s}$ is given by:

$$
F(x ; u, v):=-\frac{1}{(m-2) A_{m}} \frac{x}{|x|^{m}} H e^{\langle\omega u \omega, v\rangle} \in C^{\infty}\left(\mathbb{R}_{0}^{m}, \operatorname{End}(\mathcal{F})\right)
$$

and satisfies

$$
\mathcal{R}_{s} F(x ; u, v)=\delta(x) e^{\langle u, v\rangle} .
$$

Note that the action of the Hilbert transform $H$ on the variable $u$ is crucial. 
Proof. The reproducing kernel $e^{\langle u, v\rangle}$ has a Fischer decomposition of the form:

$$
e^{\langle u, v\rangle}=\sum_{k=0}^{\infty} \sum_{j=0}^{\infty} c_{j k} u^{j} K_{k}(u, v)
$$

for suitable constants $c_{j k}$. Recall that both the inversion operator $\mathcal{I}$ and the Hilbert operator $H$ anti-commute with the odd part of $\mathfrak{o s p}(1 \mid 2)$ (in the $u$-variable). Thus $\mathcal{I} H$ and $\mathcal{R}_{s}$ commute with $\mathfrak{o s p}(1 \mid 2)$. Hence, in view of identity (11):

$$
\mathcal{R}_{s} \mathcal{I} H e^{\langle u, v\rangle}=\sum_{k=0}^{\infty} \sum_{j=0}^{\infty} c_{j k} u^{j} \mathcal{R}_{s} \mathcal{I} K_{k}(u, v)=-(m-2) A_{m} \delta(x) e^{\langle u, v\rangle} .
$$

This proves the statement.

Recall that the Hilbert transform $H_{S}$ on the sphere is usually defined by a singular integral operator (definition 2). There exists an alternative characterization for $H_{S}$ as an integral over the projective space $S^{m-1} / \mathbb{Z}_{2}$, as we will now prove. The first step is the following:

Lemma 9. For all $H_{k}(u) \in \mathcal{H}_{k}\left(\mathbb{R}^{m}\right)$, we have that

$$
\frac{1}{A_{m}} \int_{S^{m-1}}\left(h(\omega) H_{k}(u)\right) d \omega=\frac{m-2}{2 k+m-2} H_{k}(u) .
$$

(cfr. [10] or [9] for an alternative proof based on special functions)

Proof. It suffices to consider the harmonic Fischer decomposition of the polynomial $H_{k}(x u x)$ in $\mathcal{P}_{2 k} \otimes \mathcal{H}_{k}$, with respect to the variable $x$ :

$$
H_{k}(x u x)=\sum_{p=0}^{k}|x|^{2 k-2 p} H_{2 p, k}(x, u) \quad \text { with } H_{2 p, k}(x, u) \in \mathcal{H}_{2 p} \otimes \mathcal{H}_{k} .
$$

The orthogonality of spherical harmonics on the sphere $S^{m-1}$ immediately leads to

$$
\frac{1}{A_{m}} \int_{S^{m-1}} H_{k}(x u x) d \omega=H_{0, k}(\omega, u)=H_{0, k}(0, u) .
$$

Here, we have made use of the fact that the term corresponding to $p=0$ is constant in $\omega$. To determine $H_{0, k}(0, u)$, we use the identity (9) and the fact that

$$
\Delta_{x}^{k} H_{k}(x u x)=\left(\Delta_{x}^{k}|x|^{2 k}\right) H_{0, k}(0, u)=2^{2 k} k ! \frac{\Gamma\left(k+\frac{m}{2}\right)}{\Gamma\left(\frac{m}{2}\right)} H_{0, k}(0, u) .
$$

Combining both equations indeed leads to the desired formula.

This lemma can now easily be generalized to smooth (or square integrable) functions on $S^{m-1}$ :

Proposition 9. For all $f\left(e_{u}\right) \in C^{\infty}\left(S^{m-1}\right)$, we have that

$$
\begin{aligned}
\frac{1}{A_{m}} \int_{S^{m-1}}\left(h(\omega) f\left(e_{u}\right)\right) d \omega & =\frac{m-2}{\left|m-2-2 \Gamma_{u}\right|} f\left(e_{u}\right) \\
& =\frac{m-2}{m-2-2 \Gamma_{u}}\left(H_{S} f\right)\left(e_{u}\right) .
\end{aligned}
$$


Proof. First we decompose the dense subspace $C^{\infty}\left(S^{m-1}\right)$ of $L^{2}\left(S^{m-1}\right)$ into spherical harmonics. It then suffices to apply the previous result and to use the Fischer decomposition of a spherical harmonic into spherical monogenics:

$$
H_{k}(u)=P_{k}(u)+u P_{k-1}(u), \quad P_{j}(u) \in \mathcal{M}_{j}\left(\mathbb{R}^{m}\right) .
$$

Invoking the fact that

$$
\begin{aligned}
\left(m-2-2 \Gamma_{u}\right) P_{k}(u) & =(m-2+2 k) P_{k}(u) \\
\left(m-2-2 \Gamma_{u}\right) u P_{k-1}(u) & =-(m-2+2 k) u P_{k-1}(u)
\end{aligned}
$$

proves the lemma.

This result remains valid for the extension $H$ of $H_{S}$ to $\mathcal{P}\left(\mathbb{R}^{m}, V\right)$ thus leading to the integral representation of the Hilbert operator $H$ on $\mathcal{P}\left(\mathbb{R}^{m}, V\right)$ :

Proposition 10. For all $f(u) \in \mathcal{P}\left(\mathbb{R}^{m}, V\right)$, we have that

$$
(H f)(u)=\frac{1}{A_{m}} \frac{m-2-2 \Gamma_{u}}{m-2} \int_{S^{m-1}}(h(\omega) f)(u) d \omega .
$$

Proof. This follows from the previous lemma and the fact that the action of $H, h(\omega)$ and $P_{1}$ commute with $|u|^{2}$.

\section{References}

[1] Ahlfors L.V., Old and new in Möbius groups, Ann. Acad. Sci. Fenn. Ser. A I Math., 9, pp. 93-105.

[2] Arnaudon, A., Bauer, M., Frappat, L., On Casimir's Ghost, Commun. Math. Phys. 187, pp. 429-439.

[3] Brackx, F., Delanghe, R., Sommen, F., Clifford Analysis, Research Notes in Mathematics 76, Pitman, London, 1982.

[4] Bureš, J., Sommen, F., Souček, V., Van Lancker, P., Rarita-Schwinger type operators in Clifford analysis, Journal of Funct. Anal. 185, pp. 425-456.

[5] Bureš, J., Sommen, F., Souček, V., Van Lancker, P., Symmetric analogues of Rarita-Schwinger equations, Ann. Glob. Anal. Geom. 21 No. 3, pp. 215-240.

[6] Constales, D., Sommen, F., Van Lancker, P., Models for irreducible Spin(m)-modules, Adv. Appl. Cliff. Alg. 11, pp. 271-289.

[7] Delanghe, R., Clifford analysis: history and perspective, Comp. Meth. Funct. Theory1, pp. 107-153.

[8] Delanghe, R., Sommen, F., Souček, V., Clifford analysis and spinor valued functions, Kluwer Academic Publishers, Dordrecht, 1992.

[9] Dunkl, C.F., Li J. and Ryan J., Van Lancker, P., Some Rarita-Schwinger Operators, submitted.

[10] Dunkl, C.F., Xu, Y., Orthogonal Polynomials of Several Variables, Cambridge University Press, Cambridge, 2001.

[11] Fegan, H.D., Conformally invariant first order differential operators, Quart. J. Math. 27, pp. 513-538.

[12] Frappat, L., Sciarrino, A., Sorba, P., Dictionary on Lie algebras and Superalgebras, Academic Distributors, New Delhi (2000).

[13] Gilbert, J., Murray, M.A.M., Clifford algebras and Dirac operators in harmonic analysis, Cambridge University Press, Cambridge, 1991.

[14] Gel'fand, I. M., Shilov, G. E., Generalized Functions, Vol. 1: Properties and Operations, Academic Press, New York, 1964.

[15] McIntosh, A., Clifford algebras, Fourier theory, singular integrals and harmonic functions on Lipschitz domains, in: J. Ryan (ed.), Clifford Algebras in Analysis and Related Topics, CRC Press (1996), pp. 33-88.

[16] Sommen, F., Van Acker, N., Invariant differential operators on polynomial valued functions, F. Brackx et al. (eds.), Clifford Algebras and their Applications in Mathematical Physics, Kluwer Acad. Publ., 1993, pp. 203-212. 
[17] Van Lancker, P., Rarita-Schwinger fields in the half space, Complex Variables and Elliptic Equations 51 No. 5-6 (2006), pp. 563-579. 